\section{RETROSPECTIVE ANALYSIS OF PATIENTS WITH TRANSIENT HYPOTHYROXINEMIA OF PREMATURITY: SINGLE CENTER EXPERIENCE}

Aslan Yılmaz, Ilayda Altun*, Yavuz Ozer, Hande Turan, Aydilek Dagdeviren Cakar, Hazal Cansu Acar, Yıldız Perk, Zekeriye Mehmet Vural, Oya Ercan, Olcay Evliyaoglu. Istanbul University Cerrahpasa, Cerrahpasa Medical Faculty

\subsection{6/archdischild-2021-europaediatrics.130}

Transient hypothyroxinemia of prematurity (THOP) is defined as low level of circulating thyroid hormones, despite low or normal thyroid stimulating hormone (TSH) level and detected in $35-50 \%$ of premature infants. There is no consensus on the management of THOP.Our aim was to evaluate the incidence and clinical characteristics of patients with THOP in the tertiary neonatal intensive care unit (NICU), and the rate of L-T4 treatment.

Premature newborns delivered at 24-34 wks of gestation, followed in NICU of Cerrahpasa Medical Faculty between January 2014-December 2019 were involved.

Thyroid function tests were performed between 10-20 postnatal days. Thyroid function tests were evaluated according to the gestational age references.

Among 181 infants, 52.5\% $(\mathrm{n}=95)$ were male. Mean birth weight was

$1424.85 \pm 522.58$ gr. Mean duration stay in NICU was 45.2 \pm 28.7 days. Thyroid function tests were euthyroid in $47.5 \%$ $(n=86)$ of the patients.

Hypothyroxinemia of prematurity, primary hypothyroidism and subclinical hypothyroidism were diagnosed in $45.8 \%$ $(n=83), 4.9 \% \quad(n=9)$ and $1.6 \% \quad(n=3)$, respectively. The infants were subdivided into gestational age groups as follows: 24-27 wk, $28-30$ wk, 31-34 wk. Sixty four\%(n=53) of the infants with THOP were male. Mean birth weight and gestational week were significantly lower in the hypothroxinemic group than euthyroid group. The frequency of THOP was $70 \%(\mathrm{n}=26)$ in 24-27 wk; 58\% $(\mathrm{n}=28)$ in $28-30 \mathrm{wk}$ and $35 \%(n=29)$ in $31-34$ wk. L-T4 was given to $43 \%(n=36)$ of the patients with THOP.

Treatment initiation rate was $62 \%(n=16)$ in $24-27 \mathrm{wk}$, $54 \%(\mathrm{n}=15)$ in $28-30 \mathrm{wk}$ and $17 \%(\mathrm{n}=5)$ in 31-34 wk. As the gestational week increased; incidence of THOP and the rate of treatment initiation decreased. Serum FT4 levels were lower in the treated group, but no difference was observed in terms of TSH levels. The low limit of FT4 to start treatment was determined as $0.72 \mathrm{ng} / \mathrm{dl}$ (specificity 100\%, sensitivity $33 \%)$. It was observed that the need for supportive treatment was more in the treated group.

In our study, it was observed that the prevalence of THOP increased as the gestational week and birth weight decreased. In addition, it was found that the rate of patients with low FT4 level requiring L-T4 treatment increased as the gestational week decreased and comorbid diseases increased.

\section{TRANSPORTATION OF CHILDREN BORN WITH SEVERE ASPHYXIA FROM MEDICAL INSTITUTIONS OF THE FIRST AND SECOND LEVEL OF THE PERM REGION TO A SPECIALIZED CENTER}

Yriy Kurnosov*, DV Antonov, EV Troitskaya, DV Shabunin. Perm Regional Children Clinic Hospital

10.1136/archdischild-2021-europaediatrics.131
Objective to evaluate the results of early transportation to a specialized center of children born with severe asphyxia in level I and II medical institutions.

Methods the medical histories and transport maps of 20 children born with severe asphyxia in medical institutions of the first and second level of the Perm Region and transported to the neonatal intensive care unit of the GDKB PR number 13 in 2020 were analyzed. Transportation was carried out by the resuscitation team of the department of emergency advisory medical care of the Perm regional children clinic hospital. Results 19 (95\% of children) were full - term, 1 - premature (at 35

weeks) - 1 (5\%); the average body weight was $3363.9 \pm$ $15.6 \mathrm{~g}$. All patients were on a mechanical ventilation of lungs from the birth. Taking into account the severity of the condition and the need for specialized care, these patients required emergency transportation to a specialized center in Perm. In the first day of life, $16(80 \%)$ children were taken out; 3 $(15 \%)$ were consulted on the spot due to their non-transportable condition, and later 2 children were transported when their condition was stabilized on the 2nd and 3rd days of life. The fatal outcome occurred in $2(10 \%)$ children, 1 patient died an hour after birth, 1 -on the 3 rd day. Thus, 18 patients (90\%) were transported within the first 3 days. Transportation was carried out after the assessment of the child's condition by the transporting resuscitation team after the necessary preparation and correction of treatment, mechanical ventilation of lungs, inotropic support, infusion therapy with elements of parenteral nutrition. There was no deterioration in the condition of the children during transportation. When studying the catamnesis, it was revealed that among the transported children, the mortality rate was $5.5 \%$.

Conclusion transportation to a specialized center of children born with severe asphyxia in level I and II medical institutions in the first day of life improves the prognosis and helps to reduce neonatal mortality.

\section{CORD BLOOD LEVELS OF OXIDIZED AND REDUCED GLUTATHIONE IN NEONATES EXPOSED AND NON- EXPOSED IN UTERO TO TOBACCO SMOKE}

Magdalena Chełchowska*, Joanna Gajewska, Tomasz M Maciejewski, Joanna Mazur, Mariusz Ołtarzewski, Jadwiga Ambroszkiewicz. Institute of Mother and Child

10.1136/archdischild-2021-europaediatrics. 132

Maternal tobacco smoking has been considered as an additional source of oxidant stress in pregnant women and in newborns exposed in utero, leading to perinatal and postnatal health consequences. Glutathione plays a key role in maintaining a physiological balance between prooxidants and antioxidants in human body.

Thus, we examined the relationship between glutathione status (GSH- reduced glutathione, GSSG-oxidized glutathione) and oxidative stress markers (ox-LDL- oxidized low density lipoprotein, TOC - total oxidant status) in the umbilical cord of neonates exposed and non-exposed in utero to tobacco smoke.

The study included a consecutive series of 30 healthy newborns of mothers who smoked minimum 5 cigarettes per day throughout their pregnancy, and a series of 35 neonates of mothers of similar age and age of gestation, who had never smoked and were not exposed to environmental tobacco 
smoke during pregnancy. Cord blood concentrations of biochemical markers were determined by immunoenzymatic methods and data analyses were performed using SPSS statistical software version 17.1. The study was approved by the Ethics Committee et the Institute of Mother and Child.

The newborns of smoking mothers had significantly higher concentrations of cord serum ox-LDL, TOC and GSSG $(p<0.001)$, but lower levels of GSH/GSSG ratio $(p<0.05)$ compared with newborns of non-smoking women. The levels of GSH were similar in both studied groups. Additionally, cord serum GSSG as well as TOC levels positively correlated with number of cigarettes daily consumed by the mother $(\mathrm{r}=0.49, \mathrm{r}=0.43 ; \mathrm{p}<0.05)$ and cotinine serum concentration $(\mathrm{r}=0.52, \mathrm{r}=0.43 ; \mathrm{p}<0.05)$, respectively.

Maternal smoking enhances oxidative status and depletes antioxidant potential in neonates exposed in utero to tobacco smoke. The relationship between the concentration of TOC, GSSG and the markers estimated intensity of cigarette smoking seems to confirm that the oxidant-antioxidant balance disorder in newborns is a direct result of tobacco smoke inhalation.

\section{ANTHROPOMETRICINDICATORS AND VITAMIN D LEVEL IN NEWBORNS FROM WOMEN WITH GESTATIONAL DIABETESMELLITUS}

Natalya Verisokina*, Kuryaninova Victoria, Klimov Leonid, Atanesyan Roza, Bobryshev Dmitri, Petrosyan Meline. "Stavropol State Medical University» The Ministry of Health, Stavropol, Russia

\subsection{6/archdischild-2021-europaediatrics.133}

Introduction maternal hyperglycemia during pregnancy is one of the factors of epigenetic modifications.

Objective of the study: a comparative analysis of anthropometric data and $25(\mathrm{OH}) \mathrm{D}$ level in newborns depending on the glycemic target level of the mother with GDM.

Methods 66 newborns were examined: first group - 16 (24.2\%) babies from mothers with GDM with glycemia in the III trimester of less than $5.1 \mathrm{mmol} / \mathrm{L}$; second group - 20 (30.3\%) from mothers with GDM with glycemia more than $5.1 \mathrm{mmol} / \mathrm{L}$. Control group - 30 (45.5\%) babies.

Results First and control groups: maternal glucose - 4.2 [4.04.3] $\mathrm{mmol} / \mathrm{L}$ and $4.1[3.6-4.6] \mathrm{mmol} / \mathrm{L}(\mathrm{p}>0.05)$ respectively; body weight of newborns was 3,650 [2,350-4,280] grams and $3,345[3,050-3,600]$ grams $(p>0.05)$, length 53.0 [47.0-54.0] $\mathrm{cm}$ and $51.5[50.0-53.0] \mathrm{cm}(\mathrm{p}>0.05)$, head circumference 36.0 [33.0-37.0] $\mathrm{cm}$ and $35.5[35.0-36.0] \mathrm{cm}(\mathrm{p}>0.05)$ and $25(\mathrm{OH}) \mathrm{D}$ level is $14.6[4.6-17.3] \mathrm{ng} / \mathrm{ml}$ and 14.5 [7.9-21.7] $\mathrm{ng} / \mathrm{ml}(\mathrm{p}>0.05)$ respectively.

Second group versus the control group: maternal glucose is 6.9 [5.7-7.8] $\mathrm{mmol} / \mathrm{L}(\mathrm{p}<0.001)$; weight of newborns $-3,830$ $[3,150-4,220]$ grams $(p<0.05)$; length $-53.5[50.5-55.0] \mathrm{cm}$ $(\mathrm{p}>0.05)$, head circumference $-36.0[35.0-38.0] \mathrm{cm}$ $(\mathrm{p}>0.05) ; 25(\mathrm{OH}) \mathrm{D}-6.9$ [5.7-7.8] ng/ml $(\mathrm{p}<0.05)$.

Severe deficiency of $25(\mathrm{OH}) \mathrm{D}$ in newborns from the first and second groups was detected in 5 (33.3\%) and 10 $(50.0 \%)$, deficiency - in $6(40.0 \%)$ and 7 (35.0\%), insufficiency - in $4(26.7 \%)$ and $3(15.0 \%)$ babies respectively.

Newborns from mothers with GDM with glucose more than $5.1 \mathrm{mmol} / \mathrm{L}$ had significantly higher body weight and a low level of $25(\mathrm{OH}) \mathrm{D}$ than in the control group. Babies from mothers with GDM have poorer vitamin D level than healthy newborn babies.

\section{NEONATALSEPSIS: CURRENT INFORMATION AND HOW WE ARE DOING?}

Shauna Quinn*, Daithi Kilgarrif, Noel Friesen. St John of God Midland Paediatric Department, Perth

\subsection{6/archdischild-2021-europaediatrics. 134}

Early neonatal sepsis is defined as sepsis within the first 7 days of life.

There is an incidence of $0.1-1.2 / 1000$ live births, with variation between populations. A significant reduction in GBS sepsis is recognised with introduction of intrapartum antibiotics. Predisposing risk factors include PROM, history of GBS sepsis, chorioamnionitis, prematurity and inadequate intrapartum antibiotics. The aim of this audit is to compare adequate and inadequate treatment of GBS +ve and PROM mothers and subsequent neonatal outcomes with consideration of the EOS risk calculator.

The study cohort consists of 114 neonates born at St John of God Midland between January and March. It is a retrospective review of all neonates screened during this period, with clinical data and results from Australian Clinical Labs, Infomedix and iSoft.

Of all 114 neonates screened, all were screened with a CRP and 68 with additional blood cultures. Results demonstrated one positive blood culture for S. Epidermidis, one sepsis with chorio positive growth and two presumed cases of chorioamnionitis with no growth. 50\% of those screened were treated until Mid-February, where-after there was an increase noted likely secondary to medical changeover. Amongst those screened, 66\% were empirically treated and 24\% treated following a CRP rise. We identified an issue regarding insufficient antepartum antibiotics ( $<4$ hours). 32\% of mothers were GBS +ve but only $8 \%$ received adequate antibiotics and $24 \%$ were treated inadequately.

Similarly, in PROM >18 hours only 44\% received adequate treatment despite inpatient management for $>4$ hours prior to birth. All 15 neonates born to inadequately treated mothers, were screened, two treated for CRP rises and two treated for additional risk factors.

This review highlights the importance of documentation, with inclusion of rupture of membrane and antibiotic administration times, gathering data required for the EOS calculator and liaising with the obstetric team to ensure timely administration of antibiotics. We can reduce screening and treating of neonates by optimising antepartum management or use of the EOS calculator.

\section{CHRONIC RENAL FAILURE AFTER SINGLE FETAL DEMISE IN MONOCHORIONIC TWINS - CASE REPORT}

Ana Kocijan Rebrovic*, Mirta Starcevic. University hospital centre Zagreb

\subsection{6/archdischild-2021-europaediatrics.135}

Introduction Monochorionic twins have higher rates of growth discordance, fetal loss, extreme prematurity and neonatal morbidity in general, when compared to the other types of twins. Intrauterine death of one monochorionic twin can lead to 